Original Research Paper

\title{
Amino Acid Sequence of Amylase Type Alpha, MiAmy, from Ok-Rong Mango (Mangifera indica Linn. cv. Ok-Rong)
}

\author{
${ }^{1}$ Raksmont Ubonbal, ${ }^{1,3}$ Saijai Posoongnoen, \\ ${ }^{2}$ Jureerut Daduang, ${ }^{1}$ Sompong Klaynongsruang and ${ }^{1,4}$ Sakda Daduang \\ ${ }^{1}$ Department of Biochemistry, Faculty of Science, Protein and Proteomics Research Center for Commercial and \\ Industrial Purposes (ProCCI), Khon Kaen University, Khon Kaen, 40002, Thailand \\ ${ }^{2}$ Department of Clinical Chemistry, \\ Faculty of Associated Medical Sciences, Khon Kaen University, Khon Kaen, 40002, Thailand \\ ${ }^{3}$ Chemistry Program, Faculty of Science and Technology, \\ Nakhon Ratchasima Rajabhat University, Nakhon Ratchasima, 30000, Thailand \\ ${ }^{4}$ Faculty of Pharmaceutical Sciences, Khon Kaen University, Khon Kaen, 40002, Thailand
}

Article history

Received: 10-07-2015

Revised: 20-07-2015

Accepted: 22-07-2015

Corresponding Author:

Sakda Daduang

Faculty of Pharmaceutical

Sciences, Khon Kaen

University, Khon Kaen, 40002,

Thailand

Email: sakdad@kku.ac.th

\begin{abstract}
The 594-amino acid residue sequence of $\alpha$-amylase, MiAmy, from the Ok-rong mango (Mangifera indica Linn. cv. Ok-rong), in the ripening stage, was determined through Reverse Transcription Polymerase Chain Reaction (RT-PCR). Sequence alignments and evolutionary tree analyses revealed its high similarity to plastid $\alpha$-amylase from many other plants. The sequence was revealed to have four conserved regions with catalytic amino acid residues for the active site. It was classified as a member of $\alpha$-amylase family 13 because it has an active Domain A, similar to a $(\beta / \alpha)_{8}$-barrel structure. Three-dimensional structural predictions revealed that this partial sequence completely covered all of necessary domains for amylase activity.
\end{abstract}

Keywords: $\alpha$-Amylase, Ok-Rong Mango, Amylase Family 13, Amino Acid Sequence, Mangifera indica

\section{Introduction}

$\alpha$ Amylases are classified into amylase family-13 (EC 3.2.1.1) or GH-13 which hydrolyzes $\alpha-1$, 4-Dglucosidic bonds. These proteins are found in diverse organisms and are still being characterized. $\alpha$ Amylases can be utilized for diverse applications, such as in the detergent, textile, paper, sugar and ethanol industries. For each application, it is important to understand the enzyme's specificity and function. Recently, a large number of $\alpha$ amylase proteins have been discovered in sources such as bacteria, fungi, cereals, mammal and higher plants including fruits (Torgerson et al., 1979; Kondo et al., 1980; Robyt and French, 1970; MacGregor and MacGregor, 1985; Stanley et al., 2002; SWISS-PROT, 2000; GenBank, 1982). These newly discovered enzymes vary in their structures and specificity.

Ok-rong mango (Mangifera indica Linn. cv. Okrong) is a plant indigenous to Thailand (local name; Mamuang Ok-rong). It is a large green tree that grows up to $20 \mathrm{~m}$ tall. Mangos thrive in both the subtropics and the tropics and are one of the most popular fruits both in ripe and green stages. The ripening process involves changes to several biochemical compounds which in turn cause changes in color, flavor, texture and taste. For example, in regard to flavor, soluble sugars, account for mango sweetness and accumulate through carbon supplied during both photosynthesis and starch degradation, which occur during ripening. During fruit development, starch accumulates up to $8 \%$ in the fresh pulp weight, but a low amount of soluble sugars is detected. However, during ripening, accumulated starch is rapidly converted into soluble sugars, which that can reach as high as $10 \%$ of the fresh pulp weight (Peroni Goncalves et al., 2008).

In previous studies, the amylase activity during ripening of Ok-rong mangos was significantly higher than in 30 other tested fruits (personal communication). Therefore, it is possible to discover an isoform of $\alpha$ amylase that has a high specific activity, many favorable properties and a high potential for development for industrial applications.

\section{Materials and Methods}

\section{Plant Materials}

Ok-rong mangos (Mangifera indica) in the ripening stage of were collected from Srisaket Province in 
northeastern Thailand. Pulp was ground under liquid nitrogen and stored at $-80^{\circ} \mathrm{C}$ before use.

\section{Amino Acid Sequence Determination}

Ripening Ok-rong mango tissues were disrupted by the addition of liquid nitrogen and subsequent homogenization. Total RNA was extracted using TRIzol ${ }^{\circledR}$ reagent. Then, cDNA sample were synthesized using the cDNA synthesis kit (ThermoScript ${ }^{\mathrm{TM}}$ ). For $\alpha$ amylase gene amplification, primers (Table 1) were designed from the $\alpha$ amylase sequences from other plants, Arabidopsis thaliana; AY065233, Malus domestica; AAF63939 and Citrus sinensis; XP 006483229 (Genbank). Next, specific primers were designed from our $\alpha$ amylase sequence obtained above. For PCR reactions, pre-denaturation was carried out at $94^{\circ} \mathrm{C}$ for $5 \mathrm{~min}$ followed by 35 cycles of $94^{\circ} \mathrm{C}$ for $30 \mathrm{~s}$, $55^{\circ} \mathrm{C}$ for $30 \mathrm{~s}$ and $72^{\circ} \mathrm{C}$ for $2 \mathrm{~min}$. A final extension step at $72^{\circ} \mathrm{C}$ was conducted for $10 \mathrm{~min}$. The amplicons were purified and ligated into the pGEM-T Easy vector (Promega, USA). DNA plasmids were purified and sequenced (1st Base Company, Malaysia).

The 3 ' RACE system was carried out according to the kit's instruction manual (Invitrogen, USA) using the AUAP universal primer as a reverse primer. The PCR conditions were to mix sample in the green PCR master mix kit (Fermentas, Singapore) and thermocycle at $94^{\circ} \mathrm{C}$ for $5 \mathrm{~min}$ followed by 35 cycles of $94^{\circ} \mathrm{C}$ for $30 \mathrm{~s}, 55^{\circ} \mathrm{C}$ for $30 \mathrm{~s}, 72^{\circ} \mathrm{C}$ for $2 \mathrm{~min}$ and final extension at $72^{\circ} \mathrm{C}$ for $10 \mathrm{~min}$. Then, the amplicons were purified and ligated into the pGEM-T Easy vector (Promega, USA). DNA plasmid were purified and sequenced.

\section{Bioinformatics}

Nucleotides sequences were blasted using local basic alignment search BLAST $^{\circledR}$ tools (http://blast.ncbi.nlm.nih.gov) and converted to amino acid sequences using ExPASy translation tool (http://web.expasy.org/translate). Amino acid sequence alignments were performed using the CLUSTALW program (http://ebi.ac.uk/Tools/msa/clustalw2/) using sequences from several publications and the Genbank DNA sequence database (Table 2) (Larkin et al., 2007). Phylogenetic tree and evolutionary analyses were conducted using Molecular Evolutionary Genetics Analysis $\quad$ Version $6.0 \quad$ (MEGA6; http://megasoftware.net/) (Tamura et al., 2013). Threedimensional structural predictions were launched using the SPDBV Swiss model program (http://spdbv.vitalit.ch/) (Guex and Peitsch, 1997) and the catalytic region domain was confirmed using the InterProScan Swiss model program (http://swissmodel.expasy.org/workspace/) (Zdobnov and Apweiler, 2001).

Table 1. Primers for determination of $\alpha$ amylase from Ok-rong mango ( $M$. indica) nucleotide sequences

\begin{tabular}{|c|c|c|c|}
\hline Primer pairs & Forward primer sequence & Reverse primer sequence & Expected size (bp) \\
\hline \multirow[t]{2}{*}{ FM1RM1 } & 5-GGAGGCTTGA & 5-GCGTAGACGT & 511 \\
\hline & TTTCGTTAGAG-3' & CTCTTTCTGCC-3' & \\
\hline RM2 & 5'-CAGGGTCATT & 5'-GGCCACGCG & 311 \\
\hline AUAP & GGAGGTTTC-3' & TCGACTAGTAC-3' & \\
\hline \multirow[t]{2}{*}{ FM2RM3 } & 5'-GATGCTTCT & 5'-GGCATCTTG & 435 \\
\hline & TAAAACCACC G-3' & ATTGTGGTCC-3' & \\
\hline \multirow[t]{2}{*}{ FM3RM4 } & 5'-GTTGTTCACTG & 5'-GTGCCTCAG & 500 \\
\hline & GGGAGTTTG-3' & TTTCCAAAACA AC-3' & \\
\hline FM3RM3 & 5'-GTTGTTCACTG GGGAGTTTG-3' & 5'-GGCATCTTG ATTGTGGTCC-3' & 1300 \\
\hline FM4RM3 & 5-CTTCCAGGGA AATGGATCTC-3 & 5-GGCATCTTG ATTGTGGTCC-3 & 1500 \\
\hline
\end{tabular}

Table 2. $\alpha$ Amylases included in the present study

\begin{tabular}{lllll}
\hline Plant species & Common name & Accession number & Abbreviation & $\alpha$ Amylase group \\
\hline Malus domestica & Apple & AAF63239 & MdAmyF1 & Secretory \\
Solanum tuberosum & Potato & M79328 & StAmyF1 & Secretory \\
Oryza sativa & Rice & P17654 & OsAmyF1 & Secretory \\
Hordeum vulgare & Barley & P00693 & HvAmyF1 & Secretory \\
Musa acuminata & Banana & AF533648 & MaAmyF1 & Secretory \\
Triticum aestivum & Wheat & P08117 & TaAmyF1 & Secretory \\
Zea mays & Maize & AAA50161 & ZmAmyF1 & Secretory \\
Actinidia chinensis & Kiwi & AAX33233 & AcAmyF3 & Plastid \\
Morus notabilis & Mulberry & XP_010096344 & MnAmyF3 & Plastid \\
Malus domestica & Apple & NP_001281020 & MdAmyF3 & Plastid \\
Citrus sinensis & Orange & XP_006483229 & CsAmyF3 & Plastid \\
Arabidopsis thaliana & Arabidopsis & NP5564977 & AtAmyF3 & Plastid \\
Glycine soja & Wild soybean & KHN13489 & GsAmyF3 & Plastid \\
Ricinus communis & Castor bean & XP_002520134 & RcAmyF3 & Plastid \\
Durio zibethinus Murr. cv.Mon Thong & Durian & KP164993 & DzAmyF3 & Plastid \\
Mangifera indica Linn cv.Ok-rong & Ok-rong mango & - & MiAmy & This study \\
\hline
\end{tabular}




\section{Results}

\section{MiAmy Amino Acid Sequence Determination}

Five nucleotide fragments were obtained from RTPCR techniques using 5 pairs of primers. The $3^{\prime}$ end was determined using $3^{\prime}$ RACE. There were completely overlapped (Fig. 1). Six sequence fragments were found to be part of the $\alpha$ amylase sequence. The sequence was found to be 1782 base pairs of nucleotides and share 85,83 and $79 \%$ identity with $\alpha$ amylase from durian (D. zibethinus cv. Mon thong), sweet orange (C. sinensis), mulberry (M. notabilis), respectively. The nucleotide sequence encoded 594 amino acid residues which shared 78 and $77 \%$ identity with chloroplastid $\alpha$ amylase 3 , from $D$. zibethinus and C. sinensis, respectively. It was named MiAmy.

\section{Multiple Alignment for Sequence Similarities}

Well-described sequences from previous studies (Nakajima et al., 1986; Janecek, 1992) were inputs into the CLUSTALW program and used to generate amino acid sequence alignments for $\alpha$-amylase. The large Nterminal domain of plastid $\alpha$-amylase, amino acids 447-460, was removed. The pre-defined sequences contained four conserved and semi-conserved positions, including the active site, which allowed similarities to be identified for the same regions in the novel sequence. The alignments revealed high conservation between sequence with substitutions by similar amino acid residues when compared with those from other sources including plants, mammals and bacteria. The four conserved regions are region (I) DAVLNH, (II) GWRLDFVRG, (III) GEYWD and (IV) FIENHDT. From structural studies, region II and III are suggested to be specific for the $\alpha-1,4$ glucosidic bond for $\alpha$ amylase family 13. The study of their anomeric configuration revealed their activity at one end of a polysaccharide (MacGregor et al., 2001). The four highly conserved residues are the substrate binding site; H291 and the catalytic sites; D372, E397 and D481 presented in conserved region I, II, III and IV, respectively (Fig. 2).

\section{Evolution Tree of a Amylase Ok-Rong Mango}

Phylogenetic trees with unrooted distances were calculated based on the Neighbor-Joining method (Saitou and Nei, 1987) based on the sequence similarities alignment (Fig. 2). The MiAmy was grouped into clusters along with the plastid $\alpha$ amylase members (Fig. 3). The tree reflects data from previous studies, such as a difference between conserved amino acid residues in the conserved region, especially region four. For grouping of only plastid $\alpha$ amylases, MiAmy was branched nearest to DzAmyF3 (D. zibethinus cv. Mon Thong) and CsAmyF3 (C. sinensis). This evolutionary study corresponds to the high (78 and $77 \%$ ) similarity of alignment confirming that MiAmy is an $\alpha$ amylase from plastids.

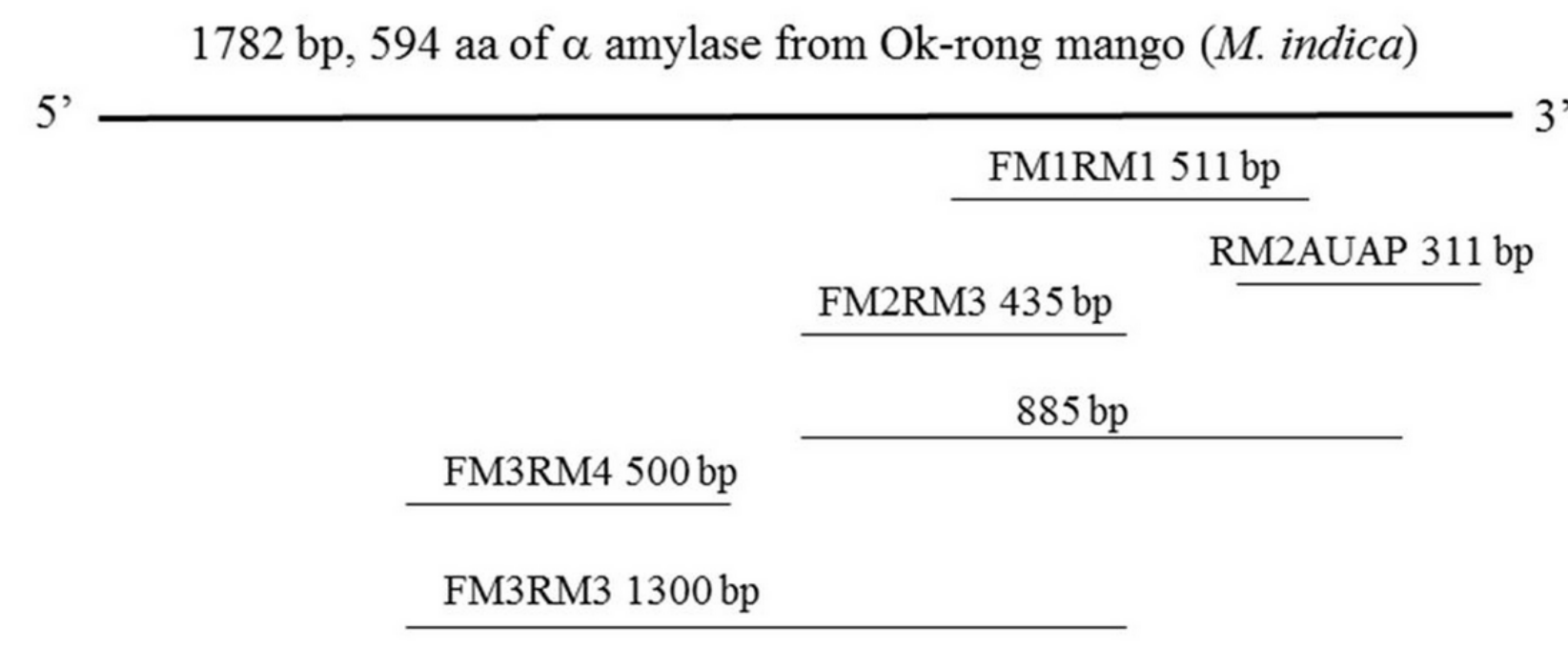

FM4RM3 $1500 \mathrm{bp}$

Fig. 1. Amino acid sequence strategies. The 594-amino acid sequence of $\alpha$-amylase was deduced from overlapping a map of the $\alpha$-amylase gene, determined by cDNA walking and 3'-RACE from Ok-rong mango (Mangifera indica) 

MdAmyr3 einany RCAnYF1 DzAmyr3 AcAnyr3 AcAnye 1 asAnyz1

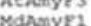
StAmyF1 OaAnyFi Mannyr1 KuAmyF1 TaAnyri

caAmyr:

MnAmyr3

Medienyry

CsAnyF

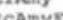

RCAHYF1
DZA-YYY 3

ACAmy 3

AcAnyr1

AtAnyrs

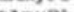

SEAnyri

idanyr:

MuAnyr2

TaAnYF 1

Madeyry

RiAmy

DzAmyr3

AcAmyY3

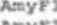

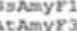

a demyza

osAmyr1

Hateyr:

GuAMYF:

تasmy

KnAstyF3

MdAmyE3

CSAmyF

tiAmy

RCAmyF1

DzAmyF 3

ACAmyF3

GSADYF1

AtAmyF3

Adtzy?

Stanyr1

OsAmyF1

AAmyr1

XuAmyF1

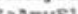

MnAmyF3

EsAmyF 3

MiAmy

RCAmyF

DzAmyr 3

ACAmyF 3

AcAmyF1

GeAmyF

AtAny? 3

MdAmyF1

StAnyE1

PSAmyE 1

Pderiris

TuAnyF 1

COFWWES-HKVORWYNELKDKARELS3LOFTVIWLPPPIESVSECOMPTDLYNLN-38Y 574 QGTWWES-SKSGRWYEEI.KSKUAELSSLOFTVIWFPPPTDSVSPQGMPPDLIYANDE-SRY 571 QGFWES-HKSGRWYMELKEKATELSSLOYSVIWLPPPTESVSPEOMPRDLYNLS-3RY 570

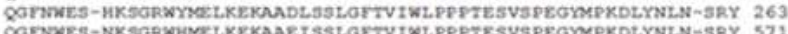

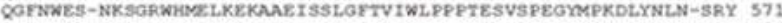

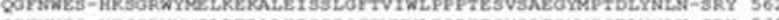

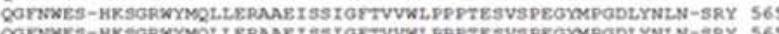

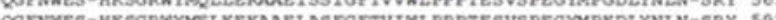
COFWES - HK. GAFNES-HKHD--

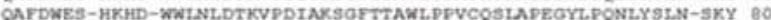
COFNWESWKENOCWYNFL NGKVDDIAUAGI THWWLPP SHSWOECGMPGRLYDLDASKY OS

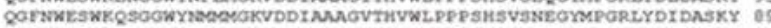

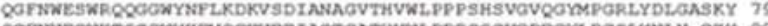
COFNEETKK QCONTKM,

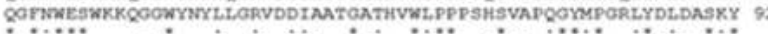

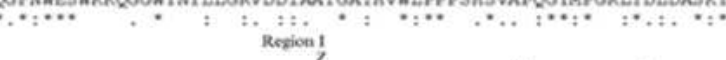

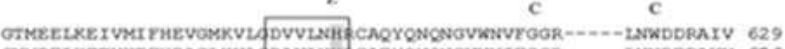

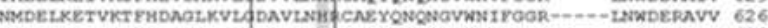

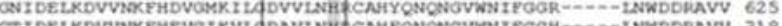

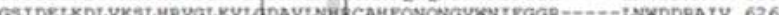

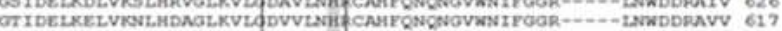

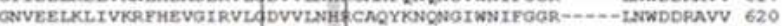

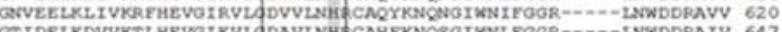

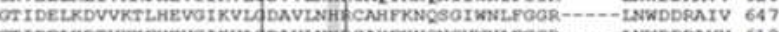

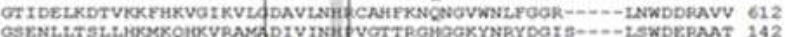

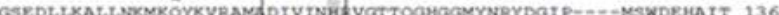

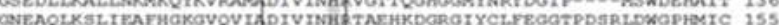

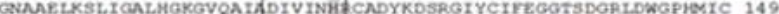

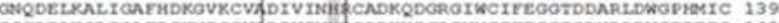
GSAADLKSLIOAFROKOIY SCVKDIVINH C CADKKDGROVYCIFECGTSDNRLDWOPDEIC 148

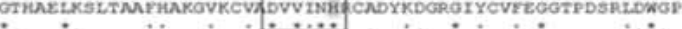

$\operatorname{coc} \mathrm{cc}$

$\frac{R}{2} \frac{1}{2} \frac{11}{2}$

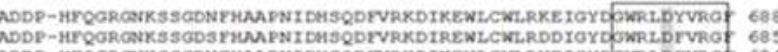

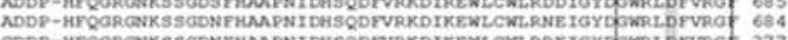

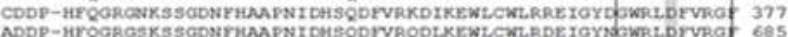

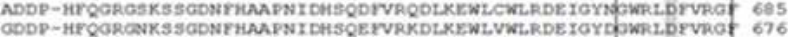

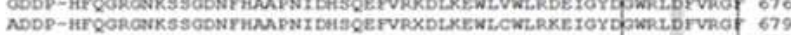

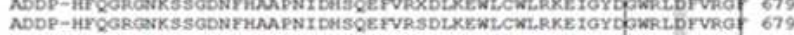

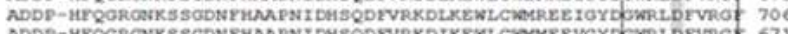

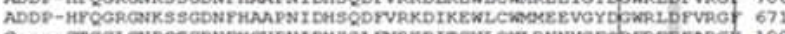

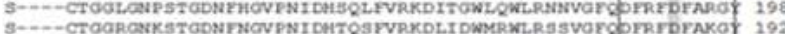
RDDP-YODGTCATPDTCADFAMAPDIDHLNKRVQRELIOWLDWLKMEDTOY WWWLLDYAKOF 214

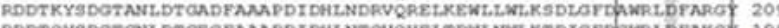
WDDTKY Y

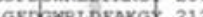
SDDTOYSN

Region III

WOGYLKDYLDSEPYFA FEEYWD-SLOYTYGEMDHMQDGHRQRIVDWINATNGT---AGA 744

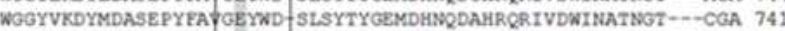

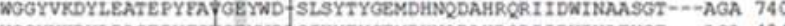

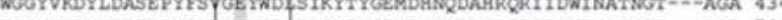
WOGYVKD YMEATEPYFA YGEYWD-SLSYTYGEMDHNQDAHRCRIIDWINATNGT---AGA 741 WGGYVKDYLDASTPYFA YGEYWD-SLSYTYGEMDHNQDAHRQRTIDWTUATNGT---AGA 732 WGGYIKDYIDASEPYFA YGEYWD-SLSXTYGEMDHNQDAHRQRIIEWINATSGT---AGA 735 WGGYIKDYIDASEPYFAY GEYWD-SLSYTYGEMDHNQDAHRQRIIEWINATSGT---AGA 735 WGGYVKDYLEASEPYFA YGEYWD-SLSYTYGENDHMQDAHRQRIVDWINATAGT---AGA 762 WGGYVKDYMDASKPYFA YGEYWD-SLSYTYGEMDYMQDAHRQRIVDWIKATSGA---AGA 727 SAKYVKEYIE CAKP I FS Y OEYWD - SCNYNGHGLDYTQDSHRQP IVNWINGTQQL---STA 25 ASKYVKEYIECAEPI FAYGEYWD-TCWYKGSNLDYNQDSHRQRI INWIDGACQL---STA 24

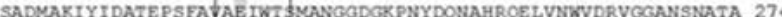

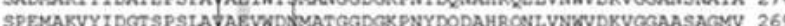

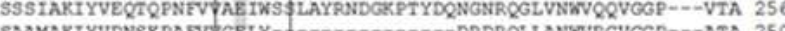
O:: : : : : :

Region IV

ww

PDVTTKGILHSALERCEYWRLSDEKGKPPGVVCWWPSRAVTFIEAHDTOSTQGHWRPPGO 804 FDVTKGILHSALDRCEYWRLSDEKGK PPGVVGWWPSPAVTIIENHDKGSTQGHWRFPGG 800

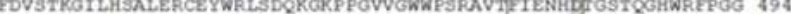

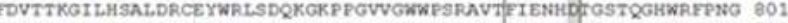

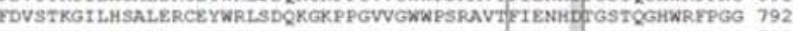
DVVITKGILHSALQRCEYWRLSDQKGKP POVVGWWPSRAVTFIENHDTGSTQGHWRFPGO 795

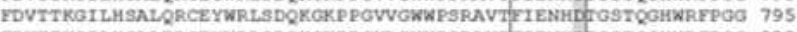

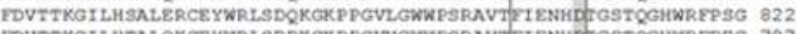

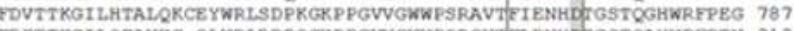

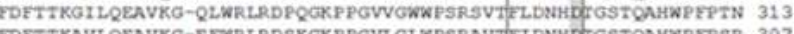

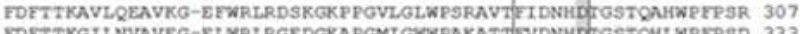

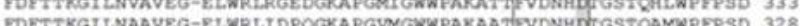

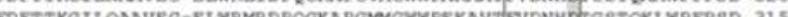
DFFTKOVT FDETTKGVLRAVQG-ELWRMKDGNGKAPGMIGWLPEKAVTRVDNHDTGSTQNSWPFPSD 328 


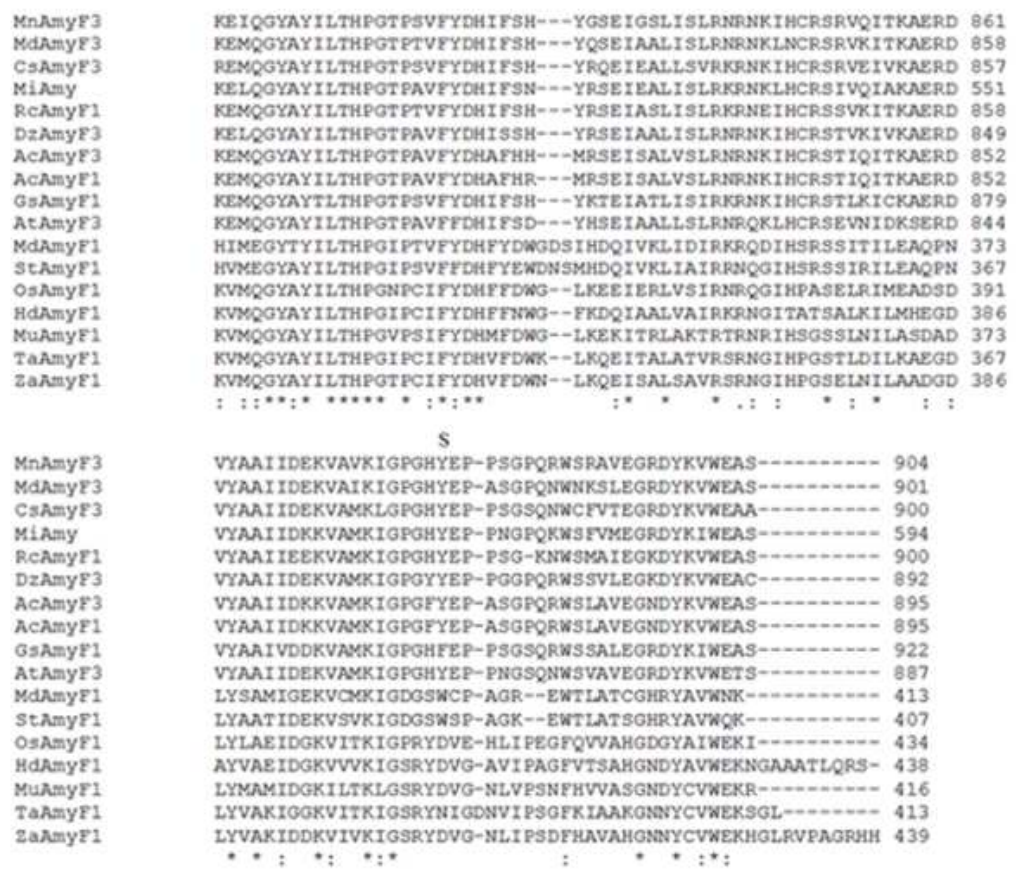

Fig. 2.A comparison of the amino acid sequences of various plant $\alpha$-amylases. Amino acid residues are shown as single letters as follows: A, Ala; C, Cys; D, Asp; E, Glu; F, Phe; G, Gly; H, His; I, Ile; K, Lys; L, Leu; M, Met; N, Asn; P, Pro; Q, Gln; R, Arg; S, Ser; T, Thr; V, Val; W, Trp; Y, Tyr. Enzyme sources are shown in Table 2. Amino acids 447-460 of plastid $\alpha$-amylases were removed. The four well-accepted regions I, II, III and IV are surrounded by rectangles. The conserved binding site and active site residues are numbered H291, D372, E397 and D481, which are present in the 4 well-accepted conserved regions I, II, III and IV, respectively. The conserved residues that are thought to be a part of the $\mathrm{Ca}^{2+}$ binding site are labeled with " $\mathrm{C}$." The residues involved in hydrogen bonding to the $\alpha$-amylase inhibitor acarbose are conserved and labeled "Z." A predicted sugar tong binding site in domain $\mathrm{C}$ is labeled "S." The names and identifying details for $\alpha$-amylases are shown in Table 2

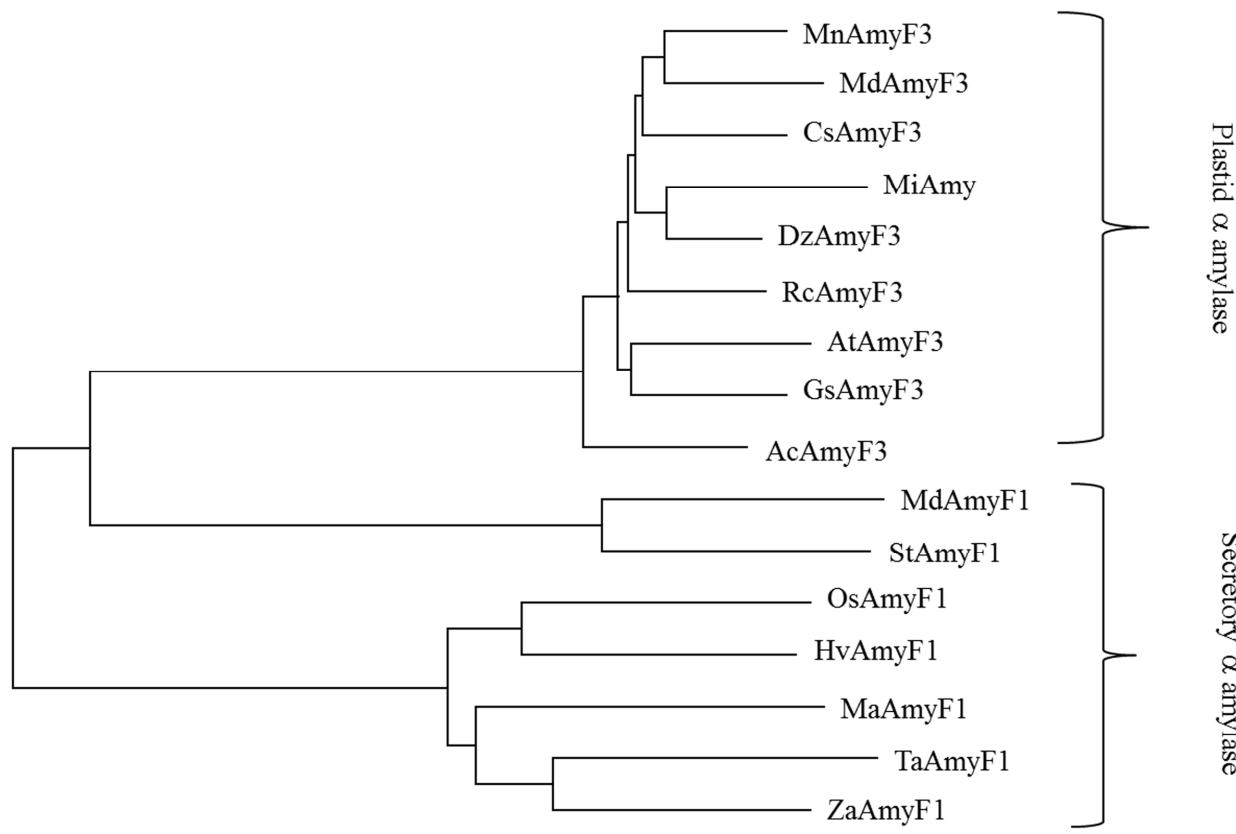

Fig. 3. The phylogenetic tree of MiAmy grouped with other $\alpha$-amylases from plants 


\begin{tabular}{|c|c|c|}
\hline & $\begin{array}{c}A \alpha 1 \\
\text { WWWWWW }\end{array}$ & \\
\hline MiAmy & TAEPEISEPSPKVSSGTGTGFEILCQGFNWESHK-SGRWYMELKEKAADLSSLGFTVIWL & 239 \\
\hline \multirow[t]{2}{*}{ HvAmyF1 } & - GHQVLFQGENWESWKQSGGWYNMMMGKVDD IAAAGVTHVWL & 64 \\
\hline & $\begin{array}{c}\mathrm{A} \alpha 2 \\
\text { WWWWWWWW }\end{array}$ & \\
\hline MiAmy & PPPTESVSPEGYMPKDLYNLN-SRYGTIDELKDVVNKFHEVGIKVLGDAVLNHRCAHFQN & 298 \\
\hline \multirow[t]{2}{*}{ HvAmyF1 } & PPPSHSVSNEGYMPGRLYDIDASKYGNAAELKSLIGALHGKGVQAIADIVINHRCADYKD & 124 \\
\hline & $\mathrm{A} \alpha 3$ & \\
\hline MiAmy & QNGVWNIFGG----HLNWDDRAVVV & 352 \\
\hline \multirow[t]{2}{*}{ HvAmyF1 } & SRGIYCI FEGGTSDGRLDWGPHMICRDDTKYSDGTANLDTGADEAAAPD IDHINDRVQRE & 184 \\
\hline & $\begin{array}{ccc}A \alpha 3 & A \beta 4 & A \alpha 4 \\
\text { Wwwwwww } & \text { ABs }\end{array}$ & \\
\hline \multirow{3}{*}{$\begin{array}{l}\text { MiAmy } \\
\text { HvAmyF1 }\end{array}$} & IKEWI QWLRRE IGYDGWRLDFVRGFWGGYNKDYLDASEPYFSVGEYWDLS IKYTYGEMDH & 412 \\
\hline & LKEWLLWLKSDLGFDAWRLDFARGYSPEMAKVYIDGTSPS LAVAEVWDNMATGGDGKPNY & 244 \\
\hline & 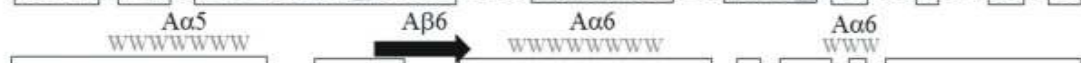 & \\
\hline \multirow{3}{*}{$\begin{array}{l}\text { MiAmy } \\
\text { HvAmyF1 }\end{array}$} & NQDAHRQRI IDWINATNGTAGA---EDVSTKGILHSALERCEYWRLSDQKGKPPGVVGWW & 469 \\
\hline & DQDAHRQNLVNWVDKVGGAASAGMVEDFTTKGILNAAVEG-ELWRLIDPQGKAPGVMGWW & 303 \\
\hline & 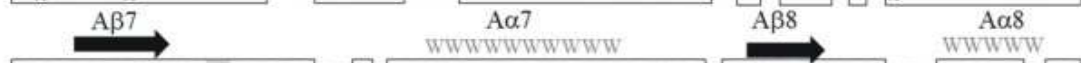 & \\
\hline \multirow{3}{*}{$\begin{array}{l}\text { MiAmy } \\
\text { HvAmyF1 }\end{array}$} & PSRAVTFIENHDTGSTQGHWRF FEGELQGYAYILTHPGTPAVFYDHIFSN-YRSEIEAL & 528 \\
\hline & PAKAATFVDNHDTGSTQAMWPEPSDKVMQGYAYILTHPGIPCIFYDHFENWGEKDQIAAL & 363 \\
\hline & $\begin{array}{c}\mathrm{Aas} \\
\text { WWWW }\end{array}$ & \\
\hline \multirow{2}{*}{$\begin{array}{l}\text { MiAmy } \\
\text { HvAmyF1 }\end{array}$} & ISLRKRNKLHCRSIVQIAKAERDVYAAI DKKVAMKIGPGHYEPPNGPQKWSFVMEGRDY & 588 \\
\hline & VAIRKRNGITATSALKILMHESDAYVAEI DGKVVVKIGSRYDVGAVIPAGEVTSAHGNDY & 423 \\
\hline MiAm & KIWEAS------ 594 & \\
\hline HvAmyF1 & AVWEKNGAAATLQRS 438 & \\
\hline
\end{tabular}

Fig. 4. Sequence alignment of MiAmy and HVAmyF1 were conducted using ClustalW alignment. Secondary structural prediction was performed using InterProScan after 201 residues of MiAmy were removed. The $\beta$-strands and $\alpha$-helices in the catalytic domain of domain $\mathrm{A}$ are labelled A $\beta 1-8$ and $\mathrm{A} \alpha 1-8$, B indicates the $\mathrm{B}$ domain and $\mathrm{C}$ denotes the C-terminal domain. Identical residues are boxed. The active site residues are shown in gray boxes

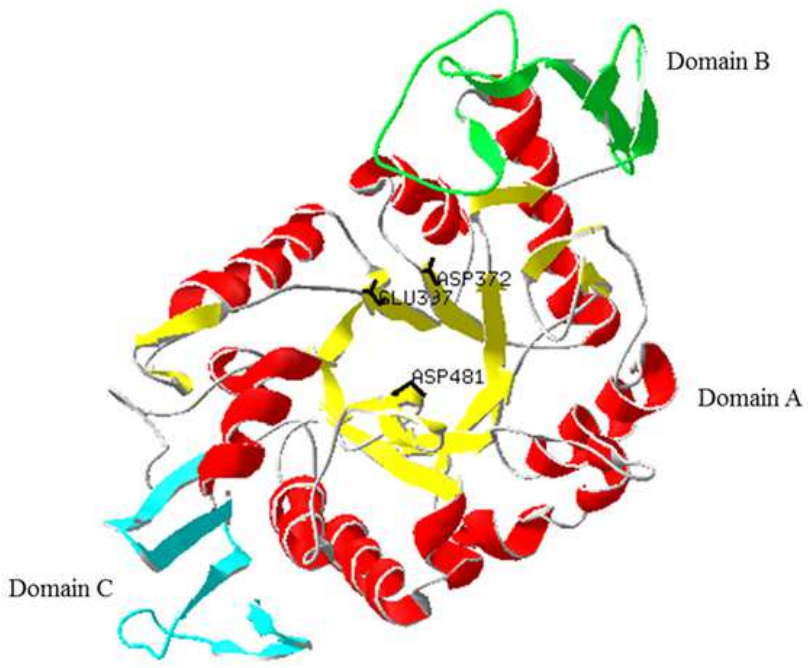

(A)

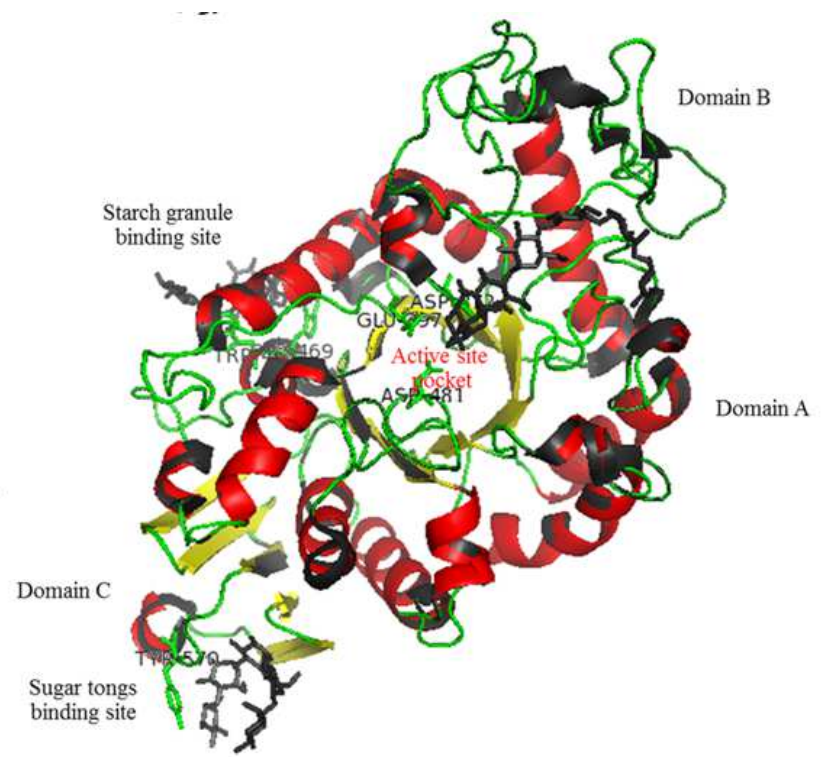

(B)

Fig. 5. Predicted 3D structure of MiAmy. A: The predicted 3D structure of MiAmy revealed domain A (red and yellow), B (green) and C (blue) containing D372, E397 and D481 as active site residues using HvAmyF1 (1RP8) as a template. B: A merged structure of $\alpha$-amylase from Ok-rong mango (color) and template (gray); the structure displayed shows the sugar tong binding site and starch granule binding site as gray sticks 


\section{$\alpha$ Amylase Secondary Structure Prediction}

InterProScan-an integration platform for the signature-recognition methods in InterPro Bioinformatics was used to determine the positions of helices and extended-chains along the polypeptide chains of plants $\alpha$ amylase. The comparison of secondary structure between MiAmy and the well study HvAmyF1 confirmed that 594 aa contained with active Domain A, substrate binding Domain B and Cterminus Domain C (Fig. 4).

\section{a Amylase Three Dimensional Structure Prediction}

For structural grouping, the predicted 3D structure of $\alpha$-amylase from Ok-rong mango was generated using the SPDBV Swiss model program and Pymol using 1RP8.pdb (crystal structure of barley alpha-amylase isoform 1) as a template (Fig. 5). The partial amino acid sequence of Ok-rong mango $\alpha$-amylase displayed $42 \%$ sequence identity with the X-ray structure of barley $\alpha$ amylase and Domain A was conserved.

\section{Discussion}

In this study, 594-amino acid MiAmy with an active domain, was confirmed to be a plastid $\alpha$ amylase and classified into the family 13 . The alignment revealed the difference between secretory $\alpha$ amylases and plastid $\alpha$ amylases. In the fourth region, the protein can be classified into two groups, FVDNHD and FIENHD for secretory and plastid $\alpha$ amylases, respectively. MiAmy region four allowed the protein to be classified into the group of plastid $\alpha$ amylase. However, the difference between VD and IE in this region does not affect the mechanistic properties. In this study, nearly all critical domains and region of MiAmy are found, which are as follows: (1) the two consecutive tryptophan residues for starch-granule binding, which correspond to W469 and W470 (Tangphatsornruang et al., 2005), (2) the conserved residues thought to be the $\mathrm{Ca}^{2+}$ binding site, (3) the residues involved in hydrogen bonding with the $\alpha$ amylase inhibitor acarbose, (4) Tyr 570 in domain C, corresponding to Tyr807 of DzAmyF3, which was suggested to be a sugar tongs surface binding site (Posoongnoen et al., 2015). Thus, the MiAmy $\alpha$ amylase gene is predicted to be valuable for further studies, including its modification.

The secondary structure of the catalytic domain of all members, including MiAmy showed the same basic $(\beta / \alpha)_{8}$ barrel, eight $\beta$ stands surrounded by eight $\alpha$ helices. Residues 202-532 of MiAmy are homologous with catalytic domains of many members of $\alpha$ amylase family 13 including residues 26-354 of MdAmyF1, 20-348 of StAmyF1, 32-372 of OsAmyF1, 26-376 of HvAmyF1,
16-354 of MaAmyF1, 26-348 of TaAmyF1, 29-367 of ZmAmyF1, 496-825 of AcAmyF3, 513-842 of MnAmyF3, 510-839 of MdAmyF3, 496-825 of CsAmyF3, 496-825 of At3AmyF, 531-860 of GsAmyF3, 510-839 of RcAmyF3 and 501-830 of DzAmyF3 (Fig. 2 ). This result correspond with the first 380 residues of secretory Taka-amylase A which constitutes the active domain necessary for $\alpha$-amylase activity (Matsuura et al., 1984). For chloroplastid $\alpha$-amylase, the active domain was characterized as the middle to the C-terminal end. Previous studies reported that an active domain, comprising amino acids 380-440, is necessary for activity of $\alpha$-amylase (MacGregor, 1988). At least 101 amino acid residues of the C-terminus could be removed without the losing enzyme activity (Yamazaki et al., 1983).

\section{Conclusion}

An amino acid sequence of $\alpha$-amylase from Okrong mango (Mangifera indica), MiAmy, was determined and classified as a plastid amylase. Bioinformatics revealed that only the amino acid residues forming the active domain are important for function and activity. In support of a previous report, 496 aa at the N-terminus of AtAmy3 was removed. The construction of recombinant shortened (390-aa) AtAmy 3 with an active domain showed equal activity when compared with the full-length native 887-aa AtAmy3 ( $\mathrm{Yu}$ et al., 2005), which supports that the identified sequence for MiAmy, containing the active domain, is sufficient for cloning and expression.

\section{Acknowledgement}

This work was financial supported by the Higher Education Research Promotion and National Research University Project of Thailand, Office of the Higher Education Commission, through the Biofuel Cluster of Khon Kaen University.

\section{Author's Contributions}

Raksmont Ubonbal: Conducted all experiments, coordinated the data analysis and drafted the manuscript.

Saijai Posoongnoen: Supervised for sequence determination and manuscript production.

Sompong Klaynongsruang: Concieved the main project idea and guide for manuscript production.

Jureerut Daduang: Guided for bioinformatics analysis.

Sakda Daduang: Designed the experiments, coordinated the study, integrated all the data, verified the result and rewrote the manuscript.

\section{Ethics}

The authors declare that there is no conflict of interest. 


\section{References}

GenBank, 1982. GenBank DNA sequence database.

Guex, N. and M.C. Peitsch, 1997. SWISS-MODEL and the Swiss-PdbViewer: An environment for comparative protein modeling. Electrophoresis, 18: 2714-2723. PMID: 9504803

Janecek, S., 1992. New conserved amino acid region of alpha-amylases in the third loop of their

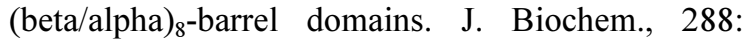
1069-1075. PMID: 1471979

Kondo, H., H. Nakatani, R. Matsuno and K. Hiromi, 1980. Product distribution in amylase-catalyzed hydrolysis of amylose. Comparison of experimental results with theoretical predictions. J. Biochem., 87: 1053-1070. PMID: 6156151

Larkin, M.A., G. Blackshields, N.P. Brown, R. Chenna and PA. McGettigan et al., 2007. Clustal W and Clustal X version 2.0. Bioinformatics, 23: 2947-2948. PMID: 17846036

MacGregor, E.A. and A.W. MacGregor, 1985. A model for the action of cereal alpha amylases on amylose. Carbohydrate Res., 142: 223-236. DOI: 10.1016/0008-6215(85)85025-4

MacGregor, E.A., 1988. Alpha-amylase structure and activity. J. Protein Chem., 7: 399-415. PMID: 3267138

MacGregor, E.A., S. Janecek and B. Svensson, 2001. Relationship of sequence and structure to specificity in the alpha-amylase family of enzymes. Biochim. Biophys. Acta, 1546: 1-20. PMID: 11257505

Matsuura, Y., M. Kusunoki, W. Harada and M. Kakudoi, 1984. Structure and possible catalytic residues of Taka-Amylase A. J. Biochem., 95: 697-702. PMID: 6609921

Nakajima, R., T. Imanaka and S. Aiba, 1986. Comparison of amino acid sequences of eleven different $\alpha$ amylases. Applied Microbiol. Biotechnol., 23: 355-360. DOI: 10.1007/BF00257032

Peroni Goncalves, F.H., C. Koike, P.R. Louro, E. Purgatto and J.R. Oliveira do Nascimento et al., 2008. Mango starch degradation. II. The binding of alpha-amylase and beta-amylase to the starch granule. J. Agric. Food Chem., 56: 7416-7421. PMID: 18656927

Posoongnoen, S., R. Ubonbal, S. Thammasirirak, J. Daduang and H. Minami et al., 2015. a-Amylase from Mon Thong durian (Durio zibethinus Murr. cv. Mon Thong)-Nucleotide sequence analysis, cloning and expression. Plant Biotechnol., 32: 1-10. DOI: $10.5511 /$ plantbiotechnology.14.1122a
Robyt, J.F. and D. French, 1970. The action pattern of porcine pancreatic alpha-amylase in relationship to the substrate binding site of the enzyme. J. Biological Chem., 245: 3917-3927. PMID: 5531189

Saitou, N. and M. Nei, 1987. The neighbor-joining method: A new method for reconstructing phylogenetic trees. Molecular Biol. Evolution, 4: 406-425. PMID: 3447015

Stanley, D., A.M. Fitzgerald, J. Kevin and F. Farnden, 2002. Characterisation of putative $\alpha$-amylases from apple (Malus domestica) and Arabidopsis thaliana. Biologia Bratislava, 57: 137-148.

SWISS-PROT, 2000. SwissProt protein sequence database. Tamura, K., G. Stecher, D. Peterson, A. Filipski and S. Kumar, 2013. MEGA6: Molecular evolutionary genetics analysis version 6.0. Molecular Biol. Evolution, 30: 2725-2729. PMID: 24132122

Tangphatsornruang, S., M. Naconsieb, C. Thammarongtham and J. Narangajavana, 2005. Isolation and characterization of an alpha-amylase gene in cassava (Manihot esculenta). Plant Physiol. Biochem., 43: 821-827. PMID: 16297635

Torgerson, E.M., L.C. Brewer and J.A. Thoma, 1979. Subsite mapping of enzymes. Use of subsite map to simulate complete time course of hydrolysis of a polymeric substrate. Archives Biochem. Biophys., 196: 13-22. DOI: 10.1016/0003-9861(79)90546-0

Yamazaki, H., K. Ohmura, A. Nakayama, Y. Takeichi and K. Otozai et al., 1983. Alpha-amylase genes (amyR2 and amyE+) from an alpha-amylasehyperproducing Bacillus subtilis strain: Molecular cloning and nucleotide sequences. J. Bacteriol., 156: 327-337. PMID: 6413492

Yu, T.S., S. Zeeman, D. Thorneycroft, D. Fulton and H. Dunstan et al., 2005. Alpha-Amylase is not required for breakdown of transitory starch in Arabidopsis leaves. J. Biological Chem., 280: 9773-9779. PMID: 15637061

Zdobnov, E.M. and R. Apweiler, 2001. InterProScan-an integration platform for the signature-recognition methods in InterPro. Bioinformatics, 17: 847-848. PMID: 11590104 